\title{
TEMPERATUREN DES BEGEHRENS ALS KONVENTIONELLE METAPHERN IM HARD-CORE-LIEBESROMAN
}

\begin{abstract}
Temperatures of desire as conventional metaphors in the new adult romance novel
The paper focuses upon the latest adult romance novels that appeared after Fifty Shades of Grey, analyses their stylistic dimensions and argues with the essay Hard-Core Romance by Eva Illouz. At first the genre is defined, above all on the basis of its medial presence (chapter 1. Genre). Furthermore temperature metaphors in two representative texts of the genre (E.L. James Fifty Shades of Grey and Sylvia Day Bared to $\mathrm{You}$ ) are analyzed as stylistic (chapter 2. Conventional Temperature Metaphors) as well as rhetorical artifacts (chapter 3. Rhetoric of Metaphor). In conclusion the question of language in the novels and its usefulness in the task described by Illouz is raised (chapter 4. A New Love Order?). The new adult romance novel seems to be a conventional cultural text of little rhetorical power, thus unable to carry important social ideas.
\end{abstract}

KEYWORDS: romance novel, genre, temperature, desire, metaphor, rhetoric

Der Sadomasochismus in der Kultur ist kein neues Phänomen und das Aufbegehren von Kritikern gegen den jüngsten Erfolg von Fifty Shades of Grey kann verwundern, betrachtet man das Buch von E.L. James und alle begleitenden Medien als rein sinnliches Vergnügen. Auch die Vermischung von BDSM (Bondage \& Discipline, Sadism \& Masochism) und Philosophie hat ihre Wurzeln schon in den Schriften de Sades, die Eva Illouz in ihrem vieldiskutierten Buch nur ungern erwähnt, als wären sie nicht der Grundstein der Idee der „,neuen Liebesordnung“. Was wahrscheinlich am meisten empört, ist die immense Faszination für BDSM (nicht zu verwechseln oder gleichzusetzen mit der Ausübung bzw. Unterstützung solcher Praktiken), sein Aufstieg in den mainstream der Popkultur, seine Pauperisierung und Verharmlosung. Wie zu Recht bemerkt werden kann, sind Krawatten und Handschellen - die eindeutigen Symbole des „neuen“ BDSM - eher wenig versprechend im Vergleich mit den Ketten, Geißeln und Klingen eines de Sade oder aber mit den mutigen Visualisierungen der Geschichte der $O$. 
Der Terminus Hard-Core Romance stammt von Eva Illouz, die im Jahre 2014 ihren Essay Hard-Core Romance. ,Fifty Shades of Grey", Best-Sellers, and Society veröffentlichte, interessanterweise nach der ein Jahr zuvor mit dem Titel Die neue Liebesordnung. Frauen, Männer und ,Shades of Grey" erschienenen deutschen Übersetzung. Die Gründe für diese eigenartige Publikationsvolte sind ohne größere Bedeutung für die vorliegenden Ausführungen, eine ähnliche Reihenfolge hatten doch auch ihre früheren Schriften Gefühle in Zeiten des Kapitalismus (2006, englisch 2007) und Warum Liebe weh tut (2011, englisch 2012). Was allerdings verwundert, sind die verschiedenen Titel, die den beiden Texten beigefügt wurden. Von der Ordnung des Liebesdiskurses und seiner geschlechtlichen Verwicklung (Frauen und Männer) zieht Illouz hier einen geraden Strich in Richtung Bestseller und Gesellschaft. Die Frage, die sie stellt, ist aber dieselbe: Wie konnte sich das mittelmäßige Buch von E.L. James als Bestseller etablieren, der schon jetzt als einer der meistgelesenen Texte des 21. Jahrhunderts bezeichnet werden kann? Der Form des Buches kann das nicht zu verdanken sein, schließlich ist es eine sehr konventionelle Liebesgeschichte, bereichert nur um Elemente von bisher als ,abnorm“ bzw. „alternativ“ angesehenen Sexualpraktiken. Den Erfolg des Buches als „kulturelle Phantasie“" , außerhalb seiner konventionellen Beschränkungen, erklärt Illouz folgendermaßen: „Die Romantrilogie bringt die Aporien heterosexueller Beziehungen verschlüsselt zum Ausdruck, bietet eine Phantasie, wie man diese Ausweglosigkeiten überwinden kann, und funktioniert letztlich als ein sexueller Selbsthilfeleitfaden.“2

Dies sollen also die Fundamente des Erfolgs sein: erstens die Darstellung der Rollenaufteilung der Geschlechter, ihrer Begrenzungen und Widersprüche, zweitens die glaubwürdige Darstellung ihrer Überwindung durch eine neue „Inszenierung der Geschlechtsidentitäten “33, hier in Form eines modernen Liebesromans mit BDSMHintergrund, drittens der Erfolg der „Erotik zum Selbermachen“4 ${ }^{\text {“4 }}$, hinter dem praktische Hinweise stehen, wie man sein Sexualleben bereichern kann. Dies sind äußerst verschiedenartige Gründe. Im ersten Fall sollte der Text als sozio-sexologische Analyse, im zweiten als „Phantasie“ (d. h. im literaturwissenschaftlichen Sinne als Narration) über eine „neue Liebesordnung“, im dritten als populärer Ratgeber gelesen werden. Diesen drei Faktoren: Wissenschaft, Literatur und Empirismus soll also der Erfolg des Buches entspringen.

Das wissenschaftliche Potenzial des Textes schätzt Illouz hoch, ihm widmet sie den größten Teil ihrer Ausführungen. Seinen literarischen Wert betrachtet sie als

\footnotetext{
${ }^{1}$ Eva Illouz: Die neue Liebesordnung. Frauen, Männer und „Shades of Grey“. Aus dem Englischen von Michael Adrian. Berlin 2013, S. 31.

${ }^{2}$ Ebd., S. 32.

${ }^{3}$ Vgl. ebd., S. 46.

${ }^{4}$ Ebd., S. 72.
} 
wenig seriös: „Shades of Grey ist schlechte Literatur“65, und es wird nicht viel mehr als dieser Satz, der letzte ihres Essays übrigens, dazu geäußert. Den wissenschaftlichen Analysen sind bei Illouz normalerweise Überlegungen zur Selbsthilfe angeschlossen (z. B. im Kapitel Sadomasochismus als Liebesutopie?), dabei um schon aus ihren früheren Schriften bekannte Thesen zum Ineinandergreifen von kapitalistischem Markt und Liebeskultur ergänzt. Ein Schwachpunkt ihres Essays ist die wenig transparente Struktur, in der manche Elemente ihrer Hauptthese (die drei Gründe für den Erfolg des Buches) vernachlässigt, andere hingegen übermäßig hervorgehoben werden. Dieser Unsicherheit entspricht eben die Wahl der Titel für die erste (deutsche) und zweite (englische) Ausgabe. Der englische Titel macht auf andere Aspekte als der deutsche aufmerksam, als ob die Autorin vor der früher angesprochenen Frauen-Männer-Problematik abrücken möchte, indem sie die Mikroperspektive (Beziehung von zwei Menschen) gegen die Makroperspektive (Gesellschaft) eintauscht.

Im vorliegenden Beitrag wird der Versuch unternommen, die von Illouz ausgelassenen Aspekte zu ergänzen. Demzufolge konzentriert er sich auf die Frage des Genres und versucht, eine Reihe von Texten, die nach dem Erfolg von Fifty Shades erschienen sind, als genologisch kohärente Gruppe darzustellen (Kapitel 1. Genre). Des Weiteren werden Temperaturmetaphern zweier repräsentativer Texte (E.L. James Fifty Shades of Grey. Geheimes Verlangen und Sylvia Day Crossfire. Versuchung) dargestellt (2. Konventionelle Temperaturmetaphern) sowie auf ihr rhetorisches Potenzial hin analysiert (3. Rhetorik der Metapher). Abschließend wird die Frage gestellt, ob die stilistische Gestaltung der Romane den von Illouz skizzierten Zwecken gewachsen ist (4. Eine neue Liebesordnung?). Als Objekte der Analyse werden Metaphern gewählt, die als Träger kultureller Bedeutungen ${ }^{6}$ den Horizont der Erzähler sowie den der Protagonist(inn)en abstecken und somit die Hauptidee des Buches darstellen könnten. Die Konzentration auf ornatus statt auf necessitas der Romanreden (wider alle Kritiken der Aufklärungsphilosophie an der angeblich entbehrlichen Metapher ${ }^{7}$ ) soll dabei gerade den verborgenen Hintergrund der Geschichte erleuchten, den Illouz als einen theoretischen und empirischen zugleich versteht und der unter dem Begriff der „neuen Liebesordnung“, d. h. einer neuen Verteilung der Kräfte in der Liebesbeziehung, zusammenzufassen wäre. ${ }^{8}$

\footnotetext{
${ }^{5}$ Ebd., S. 77.

${ }^{6}$ Vgl. Eva Kimminich: Metapher [online], <http://www.kulturenfokus.de/content/metapher> [21.09. 2015].

${ }^{7}$ Vgl. beispielsweise die Abneigung Christian Wolffs gegen die Metapher als zweideutiges Werkzeug der Sprache: Vanessa Albus: Weltbild und Metapher: Untersuchungen zur Philosophie im 18. Jahrhundert. Würzburg 2001, S. 193.

${ }^{8}$ Um die umfassende Diskussion über die Rolle der Metapher im menschlichen Denken nicht zu bemühen, soll an dieser Stelle lediglich auf die Forschungen im 20. Jh. verwiesen werden, die das kogni-
} 


\section{Genre}

Eine wenig überraschende Tatsache ist, dass der Erscheinung eines erfolgreichen Romans eine Reihe epigonaler Werke folgt, deren Urheber sich bemühen, dem Original möglichst nahe zu bleiben - sowohl im Stil als auch in allen Einzelheiten der dargestellten Welt, der Handlung sowie in der Gestaltung des Buches als Gegenstand - und trotzdem legal zu bleiben, wenn auch am Rande des Plagiats. Derartige Praktiken waren schon bei der Erscheinung der Harry Potter-Reihe zu beobachten, ebenso bei Twilight, Hunger Games, schließlich bei Fifty Shades of Grey. Es scheint, dass Serialität und beabsichtigte Epigonalität wesentliche Merkmale der Unterhaltungsliteratur des 21. Jahrhunderts sind, was sie auch in den meisten Definitionen der Postmoderne als Teil der Epoche klassifizieren ließe. Es ist eine Literatur der Wiederholung, die an einen der einfachsten Mechanismen des menschlichen Handelns appelliert: Man wählt das Bekannte; man mag, was man schon mag. ${ }^{9}$ In diesem Sinne ist sie mit den Literaturvermittlungs- und Marktmechanismen untrennbar verbunden. Ein Buch der seriellen Unterhaltungsliteratur zeichnet sich durch seine deutlichen semiotischen Bezüge aus, u. a. durch seine äußere Gestaltung und entsprechende Werbung, ungeachtet seines Inhalts und des literarischen Werts. Dadurch entsteht auch die Notwendigkeit, die bisherigen Definitionen des Genres zu revidieren und sie auch auf die mediale Präsenz des Textes auszudehnen.

Fifty Shades-Nachfolger lassen normalerweise keinen Zweifel an ihrer Abkunft. Die Buchumschläge sind mit Requisiten versehen, die schon jetzt als GreyEquipment konventionalisiert sind, dies sind: Handschellen, Stöckelschuhe, Krawatten, vornehme Betten, Unterwäsche, halbnackte Körper von Männern und Frauen sowie andere Symbole der mit Unterwerfung angehauchten Sexualität. Die Titel sollen eines der Schlüsselwörter (verführt, entfesselt, gefesselt, verboten, dunkel, gefährlich, Feuer, Schatten, Lust, Leidenschaft, Begierde, Verlockung, Versuchung usw.) enthalten, es wird auch die Tendenz sichtbar, Titel im englischen Original zu belassen, oder auch deutsche Untertitel hinzuzufügen, wenn es um Reihen und ihre einzelnen Teile geht (Rush of Love, Colours of Love, Breathless), oder wenn der englische Titel noch zu wenig illustrativ ist (Crossfire, Devoted, The Tower). Die Covers lassen sich mit der in den 90ern populär gewordenen Desire-Reihe des Harlequin-Verlags vergleichen, diesmal aber werden Rosa durch Rot/Schwarz und märchenhafte Darstellungen halbnackter, verschleierter Frauen, die oft von hinten in den Nacken geküsst werden - daher die Bezeichnung „Nackenbeißer“ - durch fotoähnliche, deutliche Zeichnungen von Körpern oder ledernen Artefakten der Lust

tive Potenzial der Metapher betonen, vor allem: George Lakoff, Mark Johnson: Metaphors We Live By. Chicago 2003 [1980].

${ }^{9}$ In dieser Hinsicht ist diese Literatur par excellence konventionell, vgl. Jonathan Culler: Structuralist Poetics. Structuralism, Linguistics and the Study of Literature. New York 1976, S. 136. 
ersetzt. ${ }^{10}$ Die Klappentexte sind sehr oft mit Verweisen auf Fifty Shades versehen und verheißen dabei ein ästhetisches Erlebnis von zumindest ähnlicher, wenn nicht weit größerer Kraft.

Auf diese Weise entsteht der Hard-Core-Liebesroman. Der Name des Genres, abgeleitet selbstverständlich von Eva Illouz (die jedoch viel mehr über ein Phänomen der Liebeskultur als über ein Genre spricht), bezieht sich auf den Ausgangspunkt - den klassischen Liebesroman mit seinen unumgänglichen Handlungsstationen (Verliebtheit - Glück - Verwicklungen - Verrat - wieder Glück - Heirat/Tod), der um ganz „moderne“ Elemente bereichert wird: Sex mit einem Schuss von Gewalt, freie Beziehungen, die Darstellung der kapitalistischen Gesellschaft (die Protagonisten arbeiten oft für große Firmen oder sind deren Chefs), Geldangelegenheiten, neueste Kommunikationsmittel, die Lebensweise von Eliten usw. Tatsächlich könnte man, dieser Spur folgend, zahlreiche ähnliche Texte, die viel früher als der Bestseller von E.L. James erschienen sind, als Vertreter des neuen Genres betrachten, angefangen mit den Schriften de Sades, stellten diese doch immer ausschweifende Amüsements reicher Libertins dar. Der Unterschied besteht jedoch darin, dass der neue HC-Liebesroman seinen Platz im so bezeichneten mainstream des Literaturbetriebs findet, somit auch eine verbreitete Anerkennung als Unterhaltungslektüre, und sich massenhaft verkauft. Es zählt das Bewusstsein der Leser, dass etwas beinahe Revolutionäres geschehen ist und dass sich ein neues Genre konstituiert. Ab jetzt wird die Geschichte der diesem Genre zuzuordnenden Literatur aus der Zeit davor und derjenigen danach bestehen. Der Auftakt ordnet sofort alle bisherigen Undeutlichkeiten wo zuvor kein Genre erkennbar war, entsteht plötzlich seine Tradition, es entstehen intertextuelle Bezüge, seine Geschichte, all dies von starken Marketingstrategien untermauert, hauptsächlich auf der Grundlage eines gemeinsamen, leicht erkennbaren Images.

Somit verwundert es nicht, dass das „,neue“, seit 2012 im öffentlichen Bewusstsein und ohne deutlichen Namen ${ }^{11}$ existierende Genre hinsichtlich seiner Struktur und verwendeten literarischen Verfahren wenig Neues zu bieten hat. Der Aufmerksamkeit der Kritiker ist es nicht entgangen, dass es sich in diesem Fall abermals um

\footnotetext{
${ }^{10}$ Interessanterweise werden dieser neuen Tendenz auch neue Ausgaben des Klassikers Geschichte der $O$ (1954) von Pauline Réage unterworfen, obwohl das Buch schon lange vor dem Erscheinen der Fifty Shades als anrüchig galt und außerhalb des mainstreams gelesen wurde, seine ursprünglichen Cover dabei weit mutiger als diejenigen des heutigen Hard-Core-Liebesromans waren. Vgl. Tanja Prokić: Celebrity Deathmatch - Shades of Grey vs. Geschichte der O., http://www.medienobservatio nen.uni-muenchen.de/artikel/literature/literature_pdf/prokic_shades.pdf (30.09.2015). Zu bemerken ist auch, dass die Desire-Reihe in letzter Zeit ihr Image verändert und im erotischen Bereich anderen Harlequin-Reihen (Blaze, New Adult, Erotic Fiction) das Feld räumt, sicherlich nicht ohne Einfluss der Fifty Shades of Grey.

${ }^{11}$ Die englische Bezeichnung ,erotic new adult romance novel” ist wenig handlich und wahrscheinlich deshalb nicht verbreitet.
} 
eine äußerst konventionelle Trivial-Liebesgeschichte (überraschende Verliebtheit, „magische“ Anziehung, Schicksal) mit mittelalterlichen bzw. romantischen Elementen (Ritterfigur mit dunklen Geheimnissen, Schloss mit Folterkammer, sozialer Aufstieg des „Mädchens aus einfachen Verhältnissen“) handelt, und dass die ,schockierenden" Elemente (Sexszenen, meistens mit Elementen von Sadomasochismus und Unterwerfung) lediglich geglättete Varietäten üblicher Beschreibungen in der Pornoliteratur darstellen. Was als relativ neu erscheinen kann, ist der Verzicht auf exzessive Plaudereien - wo bisher die beiden Liebhaber ihre Liaison detailliert erörtert haben, tritt jetzt der Sex als ihre Sprache hervor. Über Gefühle wird ebenso wenig geredet wie über Nahrung, Arbeit und Sozialversicherung, dies sind entbehrliche Beilagen in einer Welt, in der das unstillbare körperliche Begehren den Sinn des Lebens ausmacht. Somit ist auch die Sprache konventionell, benutzt abgegriffene Stilmittel, selbst wenn mit Anspruch auf künstlerischen Wert. Selten handelt es sich jedoch hierbei um Stilmittel, die einer tieferen Überlegung seitens der Leser bedürften, auch wenn sie manchmal auf ältere Kulturtexte zurückgreifen (wie beispielsweise den Ikarus-Mythos), sie sollen sofort mit Liebe und Liebemachen assoziiert werden und als solche erfüllen sie ihre Rolle ausgezeichnet. Den umfangreichsten Teil dieser Stilmittel machen Metaphern aus, die in ihrem target domain (Lakoff/Johnson) die Liebe (mit Nachdruck auf Begehren) illustrieren sollen, in ihrem source domain dagegen folgende Phänomene bemühen: Farben, Geschmack, Flug, Schweben und Fall, Schwimmen und Ertrinken, Härte und Weichheit, Musik und Singen, schließlich Temperatur. ${ }^{12}$

\section{Konventionelle Temperaturmetaphern}

Bei der kurzen Klassifikation der Temperaturmetaphern im HC-Liebesroman belassen wir es bei der klassischen, rhetorischen Aufteilung, die noch bis zur Hälfte des 20. Jahrhunderts kaum umstritten war. Aus dieser Sammlung wird u. a. die Metonymie ausgeschlossen, die in manchen Quellen als Metapher betrachtet wird. ${ }^{13}$ Diese Vereinfachung ergibt sich aus der Voraussetzung, dass nicht die Aufzählung aller möglichen Arten von Metaphern, die in den Texten des Genres zu finden sind, das Ziel des Beitrags darstellt, sondern die Schilderung der metaphorischen Dimension der Liebesbeschreibungen, die dem Text zu bestimmten rhetorischen Zwecken (wie der nächste Teil beweisen soll) auferlegt wird.

\footnotetext{
${ }^{12}$ Vgl. Lakoff/Johnson: Metaphors We Live By, S. 265.

${ }^{13}$ Obwohl Lakoff/Johnson (Metaphors We Live By, S. 35) die Metonymie nicht als Metapher ansehen, besprechen sie sie in ihrem Buch. Vgl. auch die klassische Position von Ivo Braak, Martin Neubauer: Poetik in Stichworten. Stuttgart 2007, S. 47. Hier werden die beiden Stilmittel deutlich voneinander abgegrenzt.
} 
Es ist auch zu bemerken, dass die Wirkung der Stilmittel nur im Rahmen einer gegebenen Sprachversion erörtert werden kann. Wo im Original eine Temperaturmetapher steht, kann in der Übersetzung ein für die gegebene Sprache relevantes Äquivalent eingeführt werden, das nicht unbedingt eine Temperaturmetapher sein muss.

\subsection{Personifikation}

Die Personifikation betrifft vor allem die Körperteile der Protagonistinnen und Protagonisten, mit besonderer Berücksichtigung der Geschlechtsteile. Da die Protagonist(inn)en üblicherweise zum ersten Mal in ihrem Leben derart intensive Geschlechtsakte erleben, wird der „Lebendigkeit“ und dem metaphorischen „Bewusstsein“ der Genitalien eine große Bedeutung zugemessen. Deren Fähigkeit, Temperaturzustände hervorzurufen bzw. zu verändern, soll somit als Personifikation angesehen werden, zum Beispiel:

(a) Die rhythmischen Stöße seines schweren Schwanzes sandten Wellen heißer Ekstase durch meinen gesamten Körper. (C 358) ${ }^{14}$

(b) Unter seinem Gewicht seufzte mein Herz, und heiße Sehnsucht erwärmte mein Blut. (C 205)

\subsection{Synästhesie}

Die Metapher der Sinnesvermischung hat eine lange literarische Tradition (beliebt in der Romantik und im Symbolismus), im HC-Liebesroman wird sie besonders gern verwendet für die Schilderung von metaphorischen Temperaturphänomenen, hier z. B. verbunden mit der Metaphorisierung der menschlichen Stimme:

(a) Seine Stimme klingt warm und verführerisch wie dunkler Schokoladenkaramell. (FS 32)

(b) Ich zitterte, als ich seine eiskalte Stimme hörte. (C 397)

(c) Gideons Stimme klang kühl. (C 238)

\subsection{Symbol}

Symbole eignen sich am besten zur Schilderung konventioneller Liebes- und Begehrenszustände. In der Literaturgeschichte hat sich eine große Sammlung konventioneller Temperatursymbole (hier: Feuer, Flamme, Woge) ausgebildet, aus der der HC-Liebesroman besonders gern schöpft. Der Ursprung dieser Symbole ist be-

${ }^{14}$ Die nach den Zitaten verwendeten Abkürzungen verweisen auf folgende Ausgaben: C: Sylvia Day: Crossfire. Versuchung. Aus dem Amerikanischen von Eva Malsch und Nicole Hölsken. München 2014; FS: E.L. James: Fifty Shades of Grey. Geheimes Verlangen. Deutsch von Andrea Brandl und Sonja Hauser. München 2015. 
reits kaum mehr erkennbar, sie wurden Teil der Alltagssprache, allerdings ist es sicher, dass hinter ihnen eine lange literarische Tradition steht.

(a) Flammen durchzucken meinen Körper. (FS 138)

(b) Eine warme Woge der Liebe durchflutete mich. (C 331)

(c) In meinem Körper brannte das Feuer der Lust. (C 230)

\subsection{Komplexes Symbol/Allegorie}

Der Übergang vom komplexen und stark konventionalisierten Symbol zur Allegorie ist nicht immer deutlich und kommt mittels unterschiedlicher kultureller Verträge zustande. Selbst wenn das Bild von Motte und Flamme als Metapher der Liebesanziehung immer noch am Rande der Allegorisierung bleibt, so ist der Ikarus bereits als Allegorie konventionalisiert, wenn auch meistens mit einer anderen Bedeutung als dem Liebesbegehren. Im HC-Liebesroman werden die beiden Symbole/Allegorien zur Schilderung der Wonne bzw. des Leides im „Feuer der Lust“ (siehe: 2.3. Symbol) verwendet.

(a) »Ich werde von dir angezogen wie die sprichwörtliche Motte von der Flamme.« Seine Stimme wird dunkler. »Ich begehre dich sehr, besonders jetzt, da du wieder auf deiner Lippe kaust.« Er schluckt. Mein Magen schlägt Purzelbäume - er begehrt mich... auf merkwürdige Weise, zugegeben, aber dieser attraktive, seltsame, perverse Mann will mich. »Ich glaube, es ist genau andersherum«, grummle ich. Die Motte bin ich und er das Feuer, und ich werde mir die Flügel oder was auch immer verbrennen. (FS 120)

(b) »Ich bin restlos verzaubert von dir, Christian. Überwältigt. Ich fühle mich wie Ikarus. Als würde ich der Sonne zu nahe kommen«, flüstere ich. (FS 330)

(c) Wir sind Äonen voneinander entfernt und stammen aus zwei verschiedenen Welten. Ich komme mir wie Ikarus vor, der sich an der Sonne die Flügel verbrennt und abstürzt. (FS 62)

\section{Rhetorik der Metapher}

Temperaturmetaphern können verschiedene Funktionen erfüllen, je nach dem Kontext und Register der Sprache, auf die sie sich beziehen. Ihre rhetorische Anwendung hat die Durchsetzung einer Idee zum Ziel und steht dem Konzept von Illouz nahe. Mittels rhetorischer Stilmittel (zu denen auch Metaphern gehören) könnte die Überwindung der Aporien der Beziehung dargestellt und zugleich ein Zugang zum ,verborgenen Sex-Ratgeber“ verschafft werden - wie das Begehren empfunden, stimuliert und befriedigt werden kann. Es ist dabei jedoch zu bemerken, dass Illouz die „rhetorische Kraft“ des Textes sehr begrenzt versteht: In Anlehnung an Michael Schudson stellt sie fest, die rhetorische Kraft liege ,in seinem pornographischen bzw. erotischen Inhalt ${ }^{\star 15}$, danach geht sie nicht mehr auf diesen Faktor ein

\footnotetext{
${ }^{15}$ Illouz: Die neue Liebesordnung, S. 19.
} 
und widmet ihre Aufmerksamkeit anderen Faktoren, die laut Schudson den Erfolg kultureller Artefakte in einer gegebenen Zeit bestimmen. Allerdings differenziert Schudson in seiner Untersuchung zwischen verschiedenen Formen der rhetorischen Kraft (gleichgesetzt mit effectiveness und powerfulness ${ }^{16}$ ) des Kulturartefakts: sender, audience, medium, form or format, cultural situation, message.$^{17}$ Illouz verschweigt diese Nuancierungen und lokalisiert die rhetorische Kraft im „Inhalt" des Textes (der sich in Schudsons Kategorien schwer identifizieren lässt), darüber hinaus konzentriert sie sich vor allem auf die kulturelle Resonanz des Textes, als ob diese zum Teil nicht gerade in der cultural situation und ihrer rhetorischen Kraft läge.

Indessen ist der rhetorische Kommunikationsakt weit komplexer - Schudson selbst liefert seine Hauptprämissen. Die persuasive Absicht des Senders und die Bereitschaft des Publikums, sich überzeugen zu lassen, bilden im Rahmen eines bestimmten Kulturkontextes das Wirkungsfeld für die Mitteilung, ihre Form und den Kode, der in der Mitteilung verwendet wird. ${ }^{18}$ Was uns interessiert, ist vor allem die Frage des Wie: Wie wurde der Text gestaltet? Es ist die Frage nach der Form (Genre) und der Mitteilung (message), ihrem Stil, nach der Tradition, von der sich der Stil herleitet, nach der Gestaltung, die die powerfulness des Textes in großem Maße bestimmt: „Whether an advertisement or a painting or a novel appears striking to an audience will depend very much on how skillfully the object draws from the general culture and from the specific cultural field it is a part of." ${ }^{19}$ Manches dieser Idee wurde schon angedeutet: leicht erkennbare Metaphern, Bezüge auf die Tradition, gekoppelt mit dem Anschein der künstlerischen Originalität, all das ermöglicht es dem Leser (je nach seiner literarischen Ausbildung), ein gewisses Vergnügen an der Lektüre zu finden, sich interessieren zu lassen, mehr noch: an das Potenzial des Textes als Lösung gewisser gesellschaftlicher Probleme zu glauben, in ihm sogar eine „Wahrheit“ zu finden, die sich auf das eigene Leben applizieren lässt. Deshalb erscheint es unumgänglich, einen Blick auf die rhetorische Seite des Textes zu werfen, nicht nur auf sein Was (Inhalt), sondern auch, und vor allem, auf das Wie.

\subsection{Euphemistisch-direkte Darstellung des Geschlechtsaktes}

Die Euphemisierung des Geschlechtsaktes ist ein altes Verfahren, früher motiviert durch Sitten der Epoche, Forderungen der Lesekultur, schließlich durch gesetzliche Regeln, heutzutage, da die Sitten und das Gesetz an ihrer Wirksamkeit einiges

\footnotetext{
${ }^{16}$ Michael Schudson: How Culture Works: Perspectives from Media Studies on the Efficacy of Symbols. In: Theory and Society, Vol. 18, No. 2 (1989), S. 164.

${ }^{17}$ Ebd., S. 165.

${ }^{18}$ Schudsons rhetorische „Aspekte” decken sich in groben Zügen mit Bestandteilen des Kommunikationsmodells von Jakobson, vgl. Roman Jakobson: Poetik: Ausgewählte Aufsätze 1921-1971. Hrsg. Elmar Holenstein, Tarcisius Schelbert. Frankfurt a. M. 1979, S. 88.

${ }^{19}$ Schudson: How Culture Works, S. 166.
} 
eingebüßt haben, wird sie am häufigsten als „Geschmacksfrage“ behandelt. Dass die Euphemisierung viel mehr als ornatus ist, ja für das Fortbestehen des Genres geradezu Ausmaße von necessitas annimmt, beweisen folgende Beispiele:

(a) Mit einem wortlosen Flehen um Gnade strebe ich der Sonne entgegen und verglühe in der sengenden Hitze, bevor ich falle, hinab in die Tiefen der Atemlosigkeit und der Erfüllung. Er stößt ein letztes Mal zu, dann hält er abrupt inne, als er den Höhepunkt erreicht, meine Handgelenke fest gepackt, und voller Anmut und Dankbarkeit wortlos über mir zusammensinkt. (FS 433)

(b) Gott, in diesem Augenblick fand ich Gideon heißer als die Hölle. Ich keuchte vor Aufregung, in meinem Kopf drehte sich alles. Alle möglichen Varianten tauchten vor meinem geistigen Auge auf. Meine Möse zitterte vor freudiger Erwartung. (C 321)

(c) Zum ersten Mal, seit wir zusammen waren, hielt ich keinen glühenden Stahl in den Händen, aber er wurde schnell härter und dicker, während ich ihn vorsichtig von der Wurzel zur Spitze hin streichelte. Die Furcht lauerte unter meiner Erregung, aber ich hatte mehr Angst davor, ihn zu verlieren, als davor, mit den gefährlichen Dämonen in seinem Inneren leben zu müssen. (C 356)

Die schwer identifizierbaren, quasi-poetischen, extrem euphemistischen Konventionen (sie könnten genauso gut den Harlequin-Romanen als auch den Liebesromanen aus dem 18. Jahrhundert entstammen) vermischen sich in diesen Zitaten mit direkten Ausdrücken und unverblümten, ja vulgären Schilderungen des Liebesaktes, die für die Pornoliteratur des 20. und 21. Jahrhunderts typisch sind. Aus dieser Qualität soll das Genre seine Kraft schöpfen, handelt es sich doch um eine Verschmelzung von Romantischem und Hard-Core. So erlebt der Lesende rasche Übergänge von lyrisch angehauchten Momenten zu niedrigsten Registern der Sprache, was (höchstwahrscheinlich unbeabsichtigte) komische Effekte zur Folge haben kann, wenn „zustoßen“ der „Anmut“, „geistiges Auge“ der „Möse“, „härter und dicker“ der „lauernden Furcht“ gegenübergestellt werden (vom Kontext der Reflexion über die „gefährlichen Dämonen“ ganz abgesehen). Dieses Verfahren, die Gegenüberstellung von Hohem und Niederem, war bisher aus Parodien bekannt, die die allzu hochtrabende Atmosphäre der Liebesbeschreibungen zu entladen versuchten, jetzt wird es zum Bestandteil des neuen Genres und gleichzeitig zum wichtigen Element seiner rhetorischen Kraft. Auf diese Weise kann der HC-Roman als skandalös, ja revolutionär erscheinen und den Eindruck erwecken, es handle sich um ein beispiellos mutiges künstlerisches Ereignis. Der Erfolg des Genres sollte in erster Linie von diesem Standpunkt aus analysiert werden - als Kulturartefakt, das im öffentlichen Bewusstsein die bestehenden Stilregister „dekonstruiert“.

\subsection{Darstellung des „chronischen Begehrens“}

Das Wort „chronisch“ wird üblicherweise mit Krankheit assoziiert, manchmal auch im Wirtschaftswesen als Synonym für „permanent" verwendet. Im HC-Liebesroman bezeichnet es die nie erlöschende sexuelle Anziehung der Protagonist(inn)en, 
die jeden Sexualakt als den ersten und unwiederholbaren erscheinen lässt. „Über 1800 Seiten hinweg begehrt er sie immer wieder aufs neue ${ }^{\text {‘20 }}$, schreibt Illouz, indem sie die Wandlung des Protagonisten vom Sadisten zum Liebhaber verfolgt. Da die typische Handlung der HC-Liebesromane auf Sexdarstellungen aufgebaut ist und dieses nie endende Verlangen ihren großen Teil ausmacht, müsste es auch entsprechend vielfältig dargestellt werden, um dem Eindruck der Redundanz vorzubeugen:

(a) Er hob den Kopf und sah mich an. In seinen blauen Augen brannte ein heißes, kraftvolles Feuer. »Ich sehe dich immer, mein Engel. Seit dem Augenblick, als du mich gefunden hast, sehe ich nichts außer dir «. (C 374)

(b) »Ich dachte schon, du willst Sex«, knurre ich. »Ich will immer Sex mit dir, Anastasia. Und es wärmt mir das Herz, dass es dir offenbar ebenfalls so geht«, gibt er trocken zurück. (FS 515)

(c) Er kommt langsamen Schrittes auf mich zu, selbstbewusst und sexy. Ein Gefühl heißer Lust durchzuckt mich. Er steht vor mir, sieht mir in die Augen. Er ist so verdammt sexy. (FS 129)

Diese Zitate können an beliebiger Stelle im Text ihren Platz finden, es zählt die Wiederholung. „Die Darbietung des Begehrens muss wechselseitig, zirkulär und selbsttragend sein: Ich begehre, dass dein Begehren mein Begehren begehrt und so weiter ${ }^{\text {‘21 }}$, beweist der auch von Eva Illouz (obwohl in einem anderen Kontext) zitierte Roy Brand. Die Künstlichkeit dieses Zustands, die sinnlose Zirkulation des Verlangens, der Mangel an einem sichtbaren Ausgang oder einer möglichen Veränderung, dabei das Gefühl des unermesslichen Glücks, das die Protagonist(inn)en bei ihrer ewig überraschenden Wiederentdeckung teilen, und dies noch bei sehr begrenzten sprachlichen Mitteln, lassen das ganze Konzept als Wunschdenken klassifizieren, als Paradiesvision, Pornografie (auch deren Existenz beginnt und endet mit Begehren), schließlich als Utopie, in diesem Fall eine Utopie der Liebe. Roland Barthes bemerkt: „Ist das erste Geständnis einmal abgelegt, besagt ein »Ich liebe dich « nichts mehr; es greift lediglich auf rätselhafte Weise (so leer ist sie!) die alte Botschaft wieder auf (über die diese Worte wahrscheinlich nicht hinausgehen).“22 Wenn wir das Problem von diesem Standpunkt aus betrachten, dienen alle Beteuerungen des Begehrens (u. a. „Du bist so heiß“) der Bestätigung des Unveränderlichen - obwohl die Partnerin / der Partner schon ,erobert“ ist, erlischt die Leidenschaft des Gegenübers nie, das Zeitliche, wie in einer echten Paradiesvision, wird aufgehoben, und in der beinahe magischen Formel der Wiederholung realisiert sich tatsächlich eine „neue Liebesordnung“ - die Ordnung des Liebens ohne Ende.

\footnotetext{
${ }^{20}$ Illouz: Die neue Liebesordnung, S. 44.

${ }^{21}$ Roy Brand: LoveKnowledge. The Life of Philosophy from Socrates to Derrida. New York 2012, S. 74, zitiert nach: Illouz: Die neue Liebesordnung, S. 50.

${ }^{22}$ Roland Barthes: Fragmente einer Sprache der Liebe. Übersetzt von Hans-Horst Henschen. Frankfurt a. M. 2014, S. 136.
} 


\subsection{Geschlechtliche Rollenverteilung}

Mit dem Begriff der „,neuen Liebesordnung“ meint Illouz tatsächlich eine Neuanlage von Kräften und Rollen zwischen zwei Liebenden in einer heterosexuellen Beziehung, somit auch eine Möglichkeit der Veränderung in der Ordnung der Geschlechter. Es lohnt sich zu fragen, wie diese Wandlung auf der Ebene der Sprache zustande kommt und was ihre rhetorische Kraft ausmacht.

Der geschlechtlichen Ordnung scheint auch eine eigentümliche Ordnung der Temperaturen zu entsprechen. Erstens ist sowohl die Protagonistin als auch der Protagonist zu zwei extremen Temperaturzuständen fähig (kalt und warm). Sie empfindet als Opfer und Unterdrückte, in Zuständen der Angst und der Verlassenheit Kälte. Das Gefühl der Kälteempfindung der Frau ist also ausschließlich negativ konnotiert, während ein Gefühl von Wärme bei ihr Begierde und Befriedigung, aber auch Scham und Schmerz bedeutet. Der Mann wird selten warm (am häufigsten im Sinne des umgangssprachlichen „heiß“ - als Objekt der Begierde, seltener als der Begehrende), typisch für ihn ist die Kälte, die sich vor allem in seinem „kalten Blick“ offenbart und die von Macht, Eifersucht und Missmut zeugt:

(a) Sein Oberkörper ist nackt. Ich sauge seinen Anblick auf, als wäre mein Körper vollkommen ausgedörrt und er die kühle, klare Bergquelle, die Linderung für meinen Durst verspricht. (FS 418) (b) Ich blicke in seine grauen Augen, die mich zu durchbohren scheinen. Das ist sein Dom-Blick kalt, hart, verdammt sexy, sieben Facetten der Sünde in einem einzigen Blick. Mein Mund fühlt sich staubtrocken an. Ich weiß, dass ich alles tun werde, was er von mir verlangt. (FS 566)

(c) Seine grauen Augen funkeln vor Zorn, Verlangen und purer Lust. Scheiße, allein sein Blick genügt, um mich zum Lodern zu bringen. (FS 402)

Zweitens hat nur der Protagonist ein Recht auf Widersprüche: „Brennende Lust und eiskalter Zorn. Diese beiden Gefühle kombinierte er ja gerne“ (C 391), reflektiert die Protagonistin von Crossfire. Das ist symptomatisch: Der Mann darf die ganze Palette von Temperatureindrücken, wirklichen und metaphorischen Zuständen in Anspruch nehmen, zumindest theoretisch. Im praktischen Ausmaß ist er in zwei Dimensionen gefangen, kann zwischen zwei Extremen wählen, mehr ist ihm im oben genannten Konzept des „chronischen Begehrens“ nicht vergönnt. Es wäre wenig powerful, wenn er einmal „lau“ oder ,unbestimmt“" wäre, die Zirkulation des Begehrens hätte dann keine Tragkraft, somit würde auch eine der Hauptideen des Buches die Darstellung der Liebesutopie - außer Kraft gesetzt.

Was devalviert wird, ist die Idee des Mannes als undurchsichtiger, launenhafter Machthaber, auf den der Titel Fifty Shades of Grey ursprünglich hinweisen sollte. Die fünfzig Gesichter erweisen sich bei näherem Hinsehen als ein sehr beschränktes Arsenal von Möglichkeiten. Der Mann bleibt selbstverständlich Machthaber, wenn auch ein vorhersehbarer, vor allem weil er sich seine Temperatur wählen kann, er ist es, der alle Spiele inszeniert, der die Frau zwingt, der sie in die halb-mystische Welt 
der „verbotenen Liebe“ (im Allgemeinen der Perversion) einweiht. Der Frau wird ihre Temperatur zugewiesen, entsprechende Zustände werden vom Mann eingeschaltet. Da die meisten HC-Liebesromane aus der weiblichen Ich-Perspektive erzählt werden, entstammt das Bild des Mannes, vorgeblich facettenreich, im Grunde jedoch vereinfacht „hypermaskulin“, ausschließlich ihrem „warmen Blick“.23

\section{Eine neue Liebesordnung?}

Im Hinblick auf die vorgelegten Analysen erscheinen die Ideen von Eva Illouz plausibel, allerdings nicht ohne einige Zweifel aufzuwerfen. Die „rhetorische Kraft“ des HC-Liebesromans liegt nicht ,in seinem pornographischen Inhalt“ (Illouz), sondern in seiner vollkommen konventionalisierten Sprache, die das Buch so vielen Leser(inne)n verständlich macht. Die Idee des Romans als praktischer Ratgeber bewährt sich hier - unter anderem deshalb, weil die Sprache des Romans auf konventionellen und zugleich konventionalen Metaphern basiert. Wie Robert Weninger behauptet:

Hinter jedem ,konventionalen` Konzept der Konvention steht die Grundüberzeugung, dass Wahr-
heit, Wirklichkeit, Tatsachen, Bedeutung oder Werte als Produkte von sozialen Abmachungen und
langfristigen Gewöhnungsprozessen anzusehen sind, die den Handlungsspielraum des einzelnen
zwar beschränken, ihm aber dadurch gleichzeitig in der Wirkung garantierte Mechanismen der Ver-
ständigung bereitstellen. ${ }^{24}$

Auf diese Weise tragen die Metaphern (hier Temperaturmetaphern) zur Verständigung bei, besonders im praktischen Ausmaß, helfen verstehen, „wie es ist“, egal ob lediglich der Liebesakt oder tatsächlich eine neue Ordnung der Geschlechter gemeint wird ${ }^{25}$ (,So also fühlt sich Begierde an“ (FS 80) - gesteht die Protagonistin der Fifty Shades, nicht ohne gewisse Erleichterung). Der Konvention kommt hier eine positive Bedeutung zu - als Überbrückung des Unverständlichen.

${ }^{23}$ Einen interessanten Schritt vollzog E.L. James, die der von der Protagonistin erzählten GreyTrilogie eine Art Epilog beifügte: Grey (2015), die gesamte Geschichte noch einmal erzählt, diesmal aber aus der Perspektive des Protagonisten. Abgesehen von finanziellen Antrieben der Autorin und des Verlags scheint das Publikationsverfahren aus literaturwissenschaftlicher Sicht interessant, wahrscheinlich beispiellos.

${ }^{24}$ Robert Weninger: Literarische Konventionen: Theoretische Modelle. Historische Anwendung. Tübingen 1994, S. 6.

${ }^{25}$ Vgl. Eva Illouz in ihrem Feuilleton Explaining Fifty Shades: How Bondage Solves the Problem of Modern Love für den „Spiegel Online International”: „Have you ever had sadomasochistic fantasies? If you are like me, not only have you never had any, but you even view sadomasochism as an exotic and very distant land. Assuming that most people are boringly similar to me, then it is a puzzle how Fifty Shades of Grey - a romance novel in which BDSM [...] is the central plot motif - became a phenomenal global success". [online], <http://www.spiegel.de/international/zeitgeist/eva-illouz-explains-how-fiftyshades-of-grey-solves-problems-of-love-a-843644.html> [30.09.2015]. 
Als zweifelhaft erscheint die Beweisführung beim Konzept der „neuen Liebesordnung“. Die Sprache des Romans bestätigt die bestehende Geschlechterordnung, was die Durchsetzungskraft einer neuen Ordnung der Liebesbeziehung wesentlich schwächt. Die in konventionalisierten Metaphern kodierten Rollen bewahren hier ihre Kraft. Ganz am Anfang ihrer Studie behauptet die Forscherin:

Shades of Grey [...] verkörpert den letztendlichen Triumph des weiblichen Blicks in der Kultur eines Blicks, der auf Liebe und Sexualität, auf Emotionen, auf die Möglichkeit (oder Unmöglichkeit), eine dauerhafte Liebesbeziehung mit einem Mann einzugehen, sowie auf das Ineinandergreifen von Schmerz und Lust in Liebes- und Sexualbeziehungen fokussiert ist. ${ }^{26}$

Darin soll also der zweite der drei Gründe des Romanerfolgs bestehen - die Darstellung der Überwindung eines gesellschaftlichen Problems. Es stellt sich hier jedoch die Frage, ob ein derart ambitioniertes Anliegen nicht einer neuen Sprache bedürfte, ob es in einem Text realisiert werden kann, der die alte Ordnung (der Geschlechter) nur bestätigt, und dies schon auf der Ebene des Stils. Die Rhetorik des Textes ist reaktionär und wirkt gegen das von Illouz vorgeschlagene Konzept, somit auch gegen ihre Leseweise, die an den Text große Erwartungen von gesellschaftlichem Ausmaß heranträgt.

Das kognitive (hier konventionale) Potenzial des Buches ist insofern unumstritten, als es sich um einen Sexratgeber handelt. Die vermeintliche Vision der ,neuen Liebesordnung" dagegen wurzelt in Stereotypen und Wunschdenken, ihre praktische Anwendung scheint unmöglich. Dies resultiert auch aus der fast vollkommen epigonalen stilistischen Gestaltung des Textes. Mit anderen Worten: Das Konventionale die Ankündigung einer neuen Liebesordnung, ein Vorschlag für „das Leben“, gerichtet vor allem an Frauen - wird durch das Konventionelle kritisch hinterfragt. ${ }^{27}$ Der Satz von Illouz „Shades of Grey ist schlechte Literatur“28, dem sie so wenig Bedeutung beimisst (es handle sich doch um die Idee und ihre Wirkung, nicht um die stilistische Gestaltung), hat seine Konsequenzen sowohl für den Roman, da es wenig überzeugend ist, dass er tatsächlich „,den letztendlichen Triumph des weiblichen Blicks in der Kultur" verkörpern sollte, als auch für den Essay von Illouz, in dem anhand unzureichender Argumente eine überraschend komplexe Idee forciert wird.

Wir haben mit dem Sadomasochismus begonnen, der in den mainstream der Popkultur aufgestiegen ist, widmen wir also den letzten Blick auch diesem Phänomen. Glaubt man Sigmund Freud, dass der Sadismus eine ,starke Legierung des

\footnotetext{
${ }^{26}$ Illouz: Die neue Liebesordnung, S. 9.

${ }^{27}$ Ein wichtiges Symptom dafür ist, dass die wenig anspruchsvolle konventionelle Seite des Romans sofort bemerkt und in unzähligen Parodien benutzt wurde, vgl. z. B.: ,»I play with women in my dungeon and things can get... A little hot«. »Is there no air conditioning in your dungeon? « Earl sighs. »I mean „hot” as in sexual«.” (Fanny Merkin: Fifty Shames of Earl Grey. Boston 2012, S. 98).

${ }^{28}$ Illouz: Die neue Liebesordnung, S. 77.
} 
Liebesstrebens mit dem Destruktionstrieb“29 und der Masochismus ,eine Verbindung der nach innen gerichteten Destruktion mit der Sexualität" ${ }^{\text {30 }}$ sei, dann erscheint der im HC-Liebesroman popularisierte und pauperisierte BDSM als eine unschuldige Version der Freudschen Deviation. Es fehlt die Destruktion. Der ,neue“ BDSM soll befriedigen und verbinden, ohne Ende, aber nicht mehr zerstören, was ja gewissermaßen seine letzte Konsequenz sein sollte. Die logische Folge des chronischen Begehrens ist doch der Tod. So lesen wir bei Pauline Réage in der Geschichte der O:

Die Blicke, die Hände, die Körper, die sie besudelten, die Peitschen, die sie zerfleischten, versetzten sie in einen rauschhaften Zustand der Selbstvergessenheit, der wieder in die Liebe mündete, sie vielleicht sogar in die Nähe des Todes führte. ${ }^{31}$

Die Nähe des Todes kennt der HC-Liebesroman nicht, er ist steril, selbstverliebt und in vielerlei Hinsicht trivial. Was die verächtliche Bezeichnung „Porno für Mutti“32 unbeholfen nahelegt (das Zielpublikum sollten angeblich Hausfrauen zwischen 30 und 50 sein), und was Thomas Brussig mit seiner Parole „Perversionen für alle!‘33 treffender antizipierte, verwirklicht sich im neuen Liebesroman schon auf der Ebene der sprachlichen Gestaltung, mutig genug, um große gesellschaftliche, mediale, schließlich wissenschaftliche Diskussionen zu entfesseln, und zu bescheiden, um die destruktive Tradition des Marquis de Sade wieder aufzunehmen.

\section{Literatur}

Albus, Vanessa: Weltbild und Metapher: Untersuchungen zur Philosophie im 18. Jahrhundert. Würzburg 2001.

Barthes, Roland: Fragmente einer Sprache der Liebe. Übersetzt von Hans-Horst Henschen. Frankfurt a. M. 2014.

Braak, Ivo; Neubauer, Martin: Poetik in Stichworten. Stuttgart 2007.

Brand, Roy: LoveKnowledge. The Life of Philosophy from Socrates to Derrida. New York 2012.

Brussig, Thomas: Helden wie wir. Roman. Frankfurt a. M. 2002.

Culler, Jonathan: Structuralist Poetics. Structuralism, Linguistics and the Study of Literature. New York 1976.

Day, Sylvia: Crossfire. Versuchung. Aus dem Amerikanischen von Eva Malsch und Nicole Hölsken. München 2014.

Duras, Marguerite: Der Liebhaber. Aus dem Französischen von Ilma Rakusa. Berlin 1985.

\footnotetext{
${ }^{29}$ Sigmund Freud: Das Unbehagen in der Kultur. Wien 1930, S. 93.

${ }^{30} \mathrm{Ebd}$

${ }^{31}$ Pauline Réage: Geschichte der O. Aus dem Französischen von Simon Saint Honoré. München 2006, S. 46. Vgl. auch Marguerite Duras: Der Liebhaber. Aus dem Französischen von Ilma Rakusa. Berlin 1985, S. 72: „Ich hatte ihn gebeten, es wieder und wieder zu tun. Es mir zu tun. Er hat es getan. Er hat es getan im Seim des Bluts. Und das war zum Sterben schön. Zum Sterben.”

${ }^{32}$ Illouz: Die neue Liebesordnung, S. 20.

${ }^{33}$ Thomas Brussig: Helden wie wir. Roman. Frankfurt a. M. 2002, S. 247.
} 
Freud, Sigmund: Das Unbehagen in der Kultur. Wien 1930.

Illouz, Eva: Die neue Liebesordnung. Frauen, Männer und „Shades of Grey“. Aus dem Englischen von Michael Adrian. Berlin 2013.

Illouz, Eva: Explaining "Fifty Shades": How Bondage Solves the Problem of Modern Love [online], <http://www.spiegel.de/international/zeitgeist/eva-illouz-explains-how-fifty-shades-of-grey-solvesproblems-of-love-a-843644.html>

Jakobson, Roman: Poetik: Ausgewählte Aufsätze 1921-1971. Hrsg. Elmar Holenstein, Tarcisius Schelbert. Frankfurt a. M. 1979.

James, E.L.: Fifty Shades of Grey. Geheimes Verlangen. Deutsch von Andrea Brandl und Sonja Hauser. München 2015.

Kimminich, Eva: Metapher [online], <http://www.kulturenfokus.de/content/metapher>

Lakoff, George; Johnson, Mark: Metaphors We Live By. Chicago 2003.

Merkin, Fanny: Fifty Shames of Earl Grey. Boston 2012.

Prokić, Tanja: Celebrity Deathmatch - Shades of Grey vs. Geschichte der $O$ [online], <http://www.me dienobservationen.uni-muenchen.de/artikel/literature/literature_pdf/prokic_shades.pdf>

Réage, Pauline: Geschichte der O. Aus dem Französischen von Simon Saint Honoré. München 2006.

Schudson, Michael: How Culture Works: Perspectives from Media Studies on the Efficacy of Symbols. In: Theory and Society, Vol. 18, No. 2, (1989), S. 153-180.

Weninger, Robert: Literarische Konventionen: Theoretische Modelle. Historische Anwendung. Tübingen 1994. 